\title{
The Accumulation of Profit to User and CSP Using Double Quality Guaranteed Scheme in Cloud Computing
}

\author{
Bhaskarla Sowmya ${ }^{1}$, R Lakshmi Tulasi $^{2}$ \\ ${ }^{\# 1}$ M.Tech Scholar (CSE) in Department of Computer Science \& Engineering, \\ ${ }^{\# 2}$ Prof, Head of the Department in Computer Science \& Engineering, Qis Institutute of Technology, Ongole, \\ $A P$, India.
}

\begin{abstract}
Cloud computing is a classification of web computing and with respect to request computing where shared assets and data are given to the client's on-request. Profit is the most critical variable from the cloud service provider and it is essentially dictated by the setup of a cloud profit stage under given market request. A solitary long haul leasing plan is generally used to design a cloud stage, which can't ensure the quality of administration however prompts to genuine asset squander. To beat the disadvantages of single leasing plan, Double asset RR Renting plan is composed which is the blend of both here and now and long haul leasing. Twofold asset leasing plan ensures the quality of administration as well as lessen the asset squander. In which queuing model is utilized for occupation booking. Twofold asset leasing RR conspire not just gives the Qos to the clients by utilizing load adjusting round robin calculation additionally expand profit than single leasing plan. Thirdly, a profit intensification issue is anticipated the twofold leasing arrangement and the streamlined course of action of a cloud stage is gotten by dealing with the profit help issue. Finally, a movement of calculations coordinated to break down the profit of our proposed arrange with that of the single leasing arrangement. The results exhibit that our arrangement can't simply guarantee the organization way of all requesting, furthermore get more profit than the last.
\end{abstract}

Keywords: Cloud Service Providers, Cloud computing, multi-server system, service-level agreement, waiting time, Double Renting Scheme, Profit Maximization.

\section{Introduction}

Cloud computing is rapidly turning into a successful and productive method for computing assets. Cloud computing conveys facilitated services over the Internet. Cloud computing can give the most savvy and vitality productive method for computing assets administration. Cloud computing transform's data innovation into standard products and utilities by utilizing the compensation scrutinize valuing model. A specialist co-op rents assets from the framework merchants, assembles proper multi server systems, and gives different services to clients. A consumer presents an administration demand to a specialist organization, gets the craved outcome from the specialist co-op with certain administration level understanding. At that point pays for the administration in view of the measure of the administration and the quality of the administration. A specialist co-op can manufacture distinctive multi server systems for various application areas, with the end goal that administration solicitations of various nature are sent to various multi server systems. Inferable from repetition of PC system systems and capacity system cloud may not be dependable for information, the security score is concerned. In cloud computing security is enormously enhanced as a result of a prevalent innovation security system, which is presently effectively accessible and reasonable. Applications no longer keep running on the desktop Personal Computer yet keep running in the cloud. This implies the PC does not require the preparing power or hard plate space as requested by conventional desktop programming. Capable servers and so forth are did not require anymore. The computing force of the cloud can be utilized to supplant or supplement inside computing assets. Associations no longer need to buy computing assets to deal with the limit tops. Cloud computing is rapidly turning into a successful and productive method for computing assets. By concentrated administration of assets and services, cloud computing conveys facilitated services over the Internet. Cloud computing can give the most practical and vitality proficient method for computing assets administration. Cloud computing transform's data innovation into conventional products and utilities by utilizing the compensation per-utilize evaluating model. A specialist organization rents assets from the foundation merchants, fabricates fitting multi server systems, and gives different services to clients. A buyer presents an administration demand to a specialist organization, gets the fancied outcome from the specialist organization with certain administration level understanding. At that point pays for the administration in light of the measure of the administration and the quality of the administration. A specialist organization can fabricate diverse multi server systems for various application spaces, with the end goal that administration solicitations of various nature are sent to various multi server systems. Attributable to excess of PC system systems and capacity system cloud may not be dependable for information, the security score is concerned. In cloud computing security is hugely enhanced on account of 
an unrivaled innovation security system, which is presently effortlessly accessible and reasonable. Applications no longer keep running on the desktop Personal Computer yet keep running in the cloud. This implies the PC does not require the preparing power or hard plate space as requested by conventional desktop programming. Effective servers and so forth are did not require anymore. The computing force of the cloud can be utilized to supplant or supplement interior computing assets. Associations no longer need to buy computing assets to deal with the limit crests.

\section{Related Work}

Cloud Computing and Emerging IT Platforms this paper, creator portray Cloud computing and give the auxiliary wanting to making Clouds with business division masterminded asset allotment by using progressions, for instance, Virtual Machines (VMs). Creators furthermore give bits of learning on market-based asset organization systems that join both customer driven administration and computational hazard organization to oversee Service Level Agreement (SLA)- orchestrated asset dispersion. Besides, reveal our underlying insights on interconnecting Clouds for continuously making overall Cloud exchanges and markets. By then, we show some illustrative Cloud stages, especially those made in business endeavours close by our present work towards recognizing marketsituated asset segment of Clouds as recognized in Aneka wander Cloud advancement. Additionally, creator highlight the refinement between High Performance Computing (HPC) workload moreover, Internet-based administration workload. We similarly portray a meta-game plan establishment to develop overall Cloud exchanges and publicize, and demonstrate a logical examination of furnishing 'Stockpiling Clouds' for unrivaled substance transport. Finally, creator complete up with the necessity for joining of fighting IT perfect models to pass on our 21st century vision [1]. Tradeoffs amongst Profit and Customer Satisfaction for Service Provisioning in the cloud this paper, creators use utility speculation used from money related matters likewise, develop another utility model for measuring customer satisfaction in the cloud. In light of the utility model, creators arrange an instrument to reinforce utility-based SLAs all together to alter the execution of employments and the cost of running them. We consider a framework as-an administration sort cloud arrange (e.g., Amazon EC2), where a business specialist co-op leases virtual machine (VM) events with spot costs from the cloud and grabs salary by serving its customers. Particularly, creators look at the association of administration advantage and purchaser unwaveringness. Besides, writers exhibit two booking computations that can sufficiently offer for different sorts of VM events to make trade-offs amidst advantage and purchaser dependability. Creators lead wide reenactments considering the execution data of different sorts of Amazon EC2 events and their esteem history. Creators trial comes about display that the estimations perform well over the estimations of advantage, buyer faithfulness besides, event utilize [2]. Spillage Aware Multiprocessor Scheduling this paper, spillage mindful arranging heuristics are presented that choose the best trade-off between these three techniques: DVS, processor shutdown, and finding the perfect number of processors. Exploratory outcomes got using an open benchmark set of task outlines and certified parallel applications exhibit that our philosophy decreases the total imperativeness usage by up to $46 \%$ for tight due dates and by up to $73 \%$ for nothing due dates pondered to an approach that just uses DVS. Writer moreover consider the essentialness ate up by our booking figurings to two incomparable lower limits, one for the circumstance where all processors interminably continue running at a similar repeat, and one for the circumstance where the processors can continue running at different frequencies and these frequencies may change after some time. The results exhibit that the imperativeness diminish finished by our best approach is close to these theoretical breaking points [3]. Profit drive plan for cloud services with information get to mindfulness this paper, creators address the bargain of these booking to conflict targets profit requests with the component generation of administration illustrations. Specifically, writer booking estimations attempt to extend advantage inside the pleasing level of administration quality demonstrated by the administration purchaser. Writers duties fuse (1) the change of an assessing model using processor-sharing for cloud, (2) the utilization of this evaluating model to composite services with dependence thought, (3) the progression of two courses of action of administration requesting booking figurings, and (4) the headway of a prioritization game plan for information profit wanting to enhance the advantage of information administration [4]. Vitality and Performance Management of Green Data Centers this paper, creator attempt to deal with this inadequacy by proposing an exact approach to manage open up green server homestead's advantage, i.e., wage short cost. In such way, creators unequivocally consider sensible administration level assention (SLAs) that starting at now exist between data centers and their customers. this model moreover combines diverse components, for instance, availability of neighborhood renewable compel period at server ranches and the stochastic method for server homesteads' workload. Additionally, creators propose a novel headway based advantage expansi 


\section{Service Level Agreement (SLA)}

\section{Methodology}

A service-level agreement (SLA) is a contract between customers and its service providers. A consumer sends a service request to a service provider and receives the desired result from the service provider with certain service-level agreement, then pays for the service based on the amount of the service and the quality of the service. If the service provider violates this SLA no charge is provided for the particular service. So there will be the loss of the profit. Many types of businesses use SLA. SLA adopts a price compensation mechanism to guarantee service quality and customer satisfaction. For example, China Post gives a service time commitment for domestic express mails. It promises that if a domestic express mail does not arrive within a deadline, the mailing charge will be refunded. The SLA is also adopted by many real world cloud service providers such as Microsoft Azure, Rackspace, Joyent and so on. [5]

\section{Queuing Model}

The cloud service platform is a multi-server system with a service request queue. The clouds provide resources for jobs in the form of virtual machine (VM). In addition, the users submit their jobs to the cloud in which a job queuing system such as SGE, PBS, or Condor is used. [5] In the centralized manner job scheduler schedules all jobs and assigned it to different Virtual Machines. Hence, it is one type of service request queue. For example, Condor is a specialized workload management system for compute intensive jobs and it provides a job queueing mechanism, scheduling policy, priority scheme, monitoring of resources, and management of resources. Users submit their jobs to Condor, and Condor put them into a queue, chooses when and where to run they based upon a policy. An queueing model [1] is build for our multi-server system with varying system size. And then, an optimal configuration problem of profit maximization is formulated in which many factors are taken into considerations, such as the market demand, the workload of requests, the server-level agreement, the rental cost of servers, the cost of energy consumption, and so forth. The optimal solutions are solved for two different situations, which are the ideal optimal solutions and the actual optimal solutions.

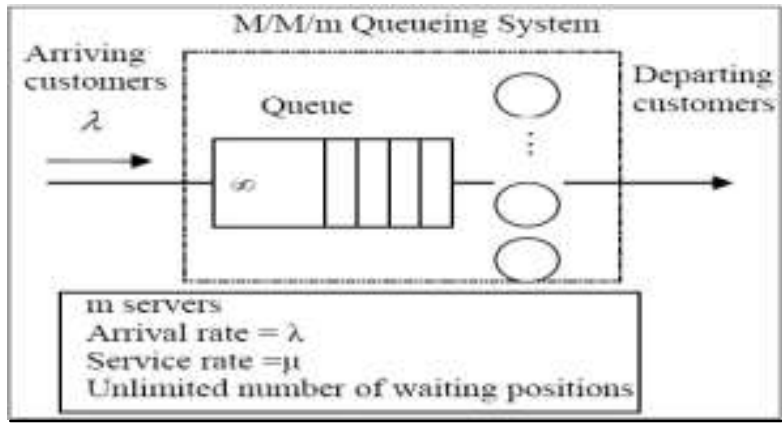

Figure 1: Queuing Model

\section{Load Balancing}

Load balancing is used to distributing a larger processing load to smaller processing nodes for enhancing the overall performance of system. In cloud computing environment load balancing is required distribute the dynamic local workload evenly between all the nodes. [8]

Load balancing is used to allocate computing resource to achieve a high satisfaction of user and proper utilization of resources. High utilization of resources and proper balancing of load helps in reducing resource consumption. It helps in implementing fail over, scalability, and avoids bottlenecks.

- Load balancing is a technique that helped networks and resources by providing a Maximum throughput with minimum response time. Load balancing [8] is divide the traffic between all servers, so data can be sent and received without any delay with load balancing.

- In cloud environment many algorithms are available that helps in proper traffic Loaded between all available servers. Most of them can be applied in the cloud environment with suitable verifications. In cloud computing environment load balancing algorithms is split into two main groups: first algorithm type is Batch mode heuristic scheduling algorithms (BMHA) and second is online mode heuristic algorithms. In BMHA Jobs are combined together when they are arriving in the system. The BMHA scheduling algorithm will start after a fixed period of time.

- Min algorithm, First Come First Served Scheduling algorithm (FCFS), Max Min algorithm, Round Robin scheduling algorithm (RR), and are the examples of BMHA based algorithms.[8]

- In our daily life example websites are the example of load balancing. Users could face many problems without Load balancing like delays, timeouts and long system responses.[8] 


\section{System Architecture}

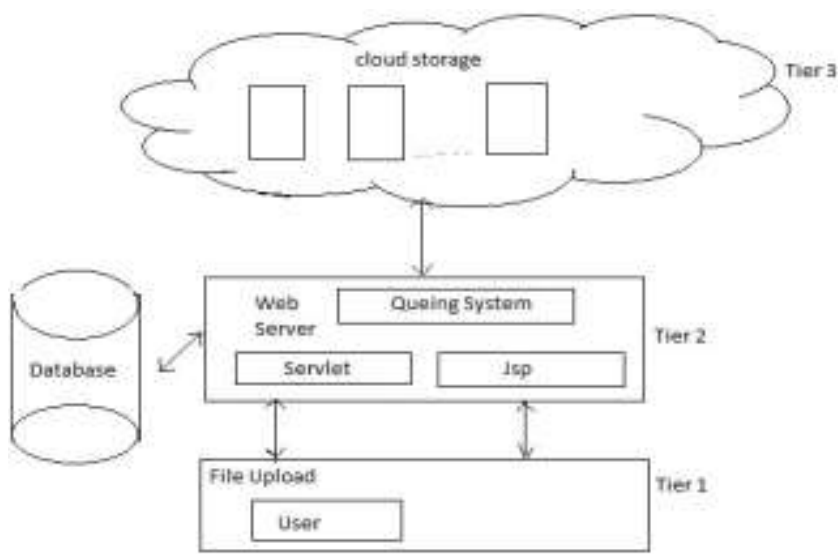

Figure 2: System Architecture

A novel renting scheme for service providers is proposed, which can satisfy quality-of-service requirements as well as obtain more profit. A double resource renting RR scheme which is also called as double quality guaranteed RR Scheme (DQG-RR) is proposed for service providers. It combines long-term renting with short-term renting, which not only satisfy quality-of-service requirements under the varying system workload, but also reduce wastage of resources. we are going to implement and improve QOS of cloud service provider by balancing workload of VMs. Whenever workload of one VM increased then the request get transfer and processed by another VM. We are going to develop load balancing algorithm to maximize the profit of our cloud. A multi-server system adopted is modeled as an $\mathrm{M} / \mathrm{M} / \mathrm{m}+\mathrm{D}$ queuing model and the performance indicators such as the average service charge, the ratio of requests that need short term servers are analyzed. The optimal configuration problem of service providers for profit maximization is formulated and two kinds of optimal solutions, i.e., the ideal solutions and the actual solutions, are obtained respectively.

A few major goals of load balancing algorithms:

- Cost effectiveness: primary aim is to achieve an overall improvement in system performance at a reasonable cost.

- Scalability and flexibility: the distributed system in which the algorithm is implemented may change in size or topology. So the algorithm must be scalable and flexible enough to allow such changes to be handled easily.

- Priority: prioritization of the resources or jobs need to be done on beforehand through the algorithm itself for better service to the important or high prioritized jobs in spite of equal service provision for all the jobs regardless of their origin.

\section{Cloud Renting Phases}

In this paper various provable cloud renting phasing are discussed.

\section{A. Cloud Subscription}

First phase of cloud service outsourcing process is Cloud Subscription. Cloud computing define by a type of outsourcing services. In which the search the information in a server. It allowing users to access technology-enabled services from the Internet in the cloud without knowledge of, or control over the technologies behind these servers. This Cloud subscriptions required to the user for storage and process. And user subscribe cloud service based on the requirements.

\section{B. Double-Quality-Guaranteed (DQG)}

In this phase, Heterogeneous double-Quality- Guaranteed (DQG) resource renting scheme which combine-s long-term with short-term renting. The main computing capacity is provided by the long- term rented servers because of their low price. The short-term rented servers will provide the extra capacity in peak period. The proposed DQG scheme adopts the traditional queuing discipline. For each service request entering by the system, and the system records its waiting time. The requests are assigned by the executed on the long- term rented servers in the order of arrival times. And the profit maximization problem in a homogeneous cloud environment. 


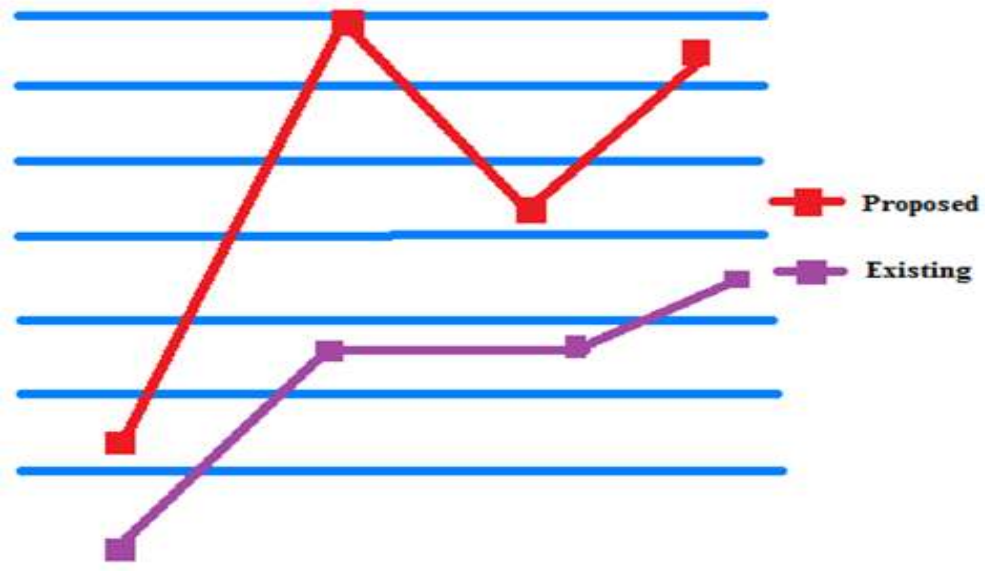

Figure 3. DQG Renting Scheme of Throughput Analysis

\section{DQG in heterogeneous}

In this phase, DQG is implemented in heterogeneous environment. In order to guarantee the QOS requests and maximize the profit of service providers, this paper has proposed a novel Double-QualityGuaranteed (DQG) renting scheme for service providers. In addition, series of calculations are conducted to compare the profit obtained by the DQG renting scheme with the Single-Quality-Unguaranteed (SQU) renting scheme. The results show that outperforms the SQU scheme in terms of both service quality and profit. The analysis of a heterogeneous environment is much more complicated in the cloud process. So heterogeneous in Double- Quality-Guaranteed (DQG) is provide the Maximization Scheme with Guaranteed Quality of Service in Cloud Computing.

\section{Predict Efficient QOS Service Provider in client}

In this phase the service providers considered as cloud brokers because they can play an important role in between cloud customers and infrastructure providers. QOS is predict the efficient cloud service to the client. In QOS provides the basic hardware and software facilities. The cloud service provider rents resources from the infrastructure providers. The cloud provide resources for job and set of services in the form of virtual machine (VM). The infrastructure service providers for profit maximization is formulated and two kinds of resource renting schemes, e.g., long-term renting scheme, short-term renting scheme. In general, the rental price of longterm renting scheme is much cheaper than that of short-term renting scheme.

\section{Quality-Guaranteed Scheme}

The custom single resource renting scheme cannot guarantee the quality of all requests but wastes a great amount of resources due to their uncertainty of system workload. To overcome the weakness, we propose a double renting scheme as follows, which cannot guarantee the quality of service completely but also can reduce the resource waste greatly.

\section{A. Proposed Scheme}

In this section, first we propose the double-Quality- Guaranteed (DQG) resource renting scheme which combines long-term with short-term renting. The main computing capacity is provided by the long- term rented servers because of their low price. The short-term rented servers will provide the extra capacity in peak period. Since the requests with waiting time are all assigned to temporary services, it is apparent all temporary services are rented for all requests whose waiting time are equal to the deadline and are charged based on the workload according to the SLA [9]. Hence, the cost and revenue of the service provider will be increases. However, the cost increases as well as due to the temporarily rented services. Moreover, the amount of renting cost spent in temporary services is determined by the computing capacity of the long- term rented multi-server system. The maximizing the profit first should understand the cost and revenue using our scheme. Since minimizing the cost is the key issue for profit maximization. Next, the trade-off between the long- term rental cost and short-term rental cost is considered. Optimal problem is formulated in the following to get the, long-term optimal configuration such that the profit 


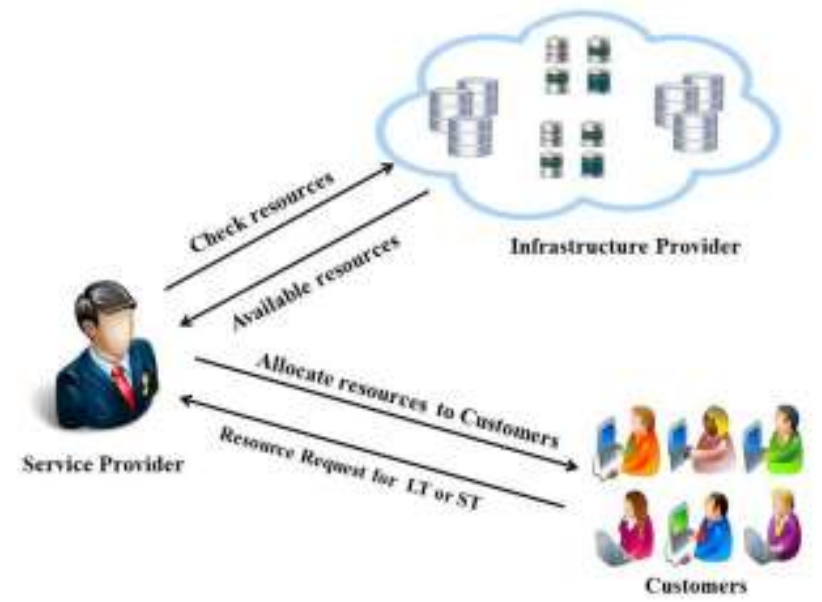

Figure 4. Proposed Architecture diagram

\section{Proposed Algorithm}

\section{Double Quality Guaranteed Scheme}

Step 1: A multiserver system is running with $\mathrm{m}$ servers and waiting for events.

Step 2: queue $Q$ is initialized as empty.

Step 3: Event-when service request arrives and server is available then assign service request to that server.

Step 4: if server is not available then put service request at end of the queue $\mathrm{Q}$ and record its waiting time.

Step 5: Event-when server becomes idle and queue is empty then waiting for new service request.

Step 6: if queue is not empty then take first service request and assign to that idle server.

Step 7: Event - deadline of a request is achieved then rent a temporary server to execute the request and release that server when request is completed.

\section{Mathematical Module}

$\mathrm{S}=(\mathrm{I}, \mathrm{F}, \mathrm{O}, \mathrm{C})$

$\mathrm{S}=$ System

$\mathrm{I}=$ Set of inputs $=$ user requests, package

$\mathrm{O}=$ Generated output $=$ Plan granted

$\mathrm{F}=$ Set of functions

$\mathrm{F} 1=$ Cloud User login, registration, upload file, view policy terms, choose package, cost.

F2 = BSP login, view user requests and accept them, publish infrastructure services and view graph modulations. F3 = ISP login, provide infrastructure services, view broker requests, maintain users.

\section{Venn diagram}

ISP: Infrastructure service provider

BSP: Business service provider

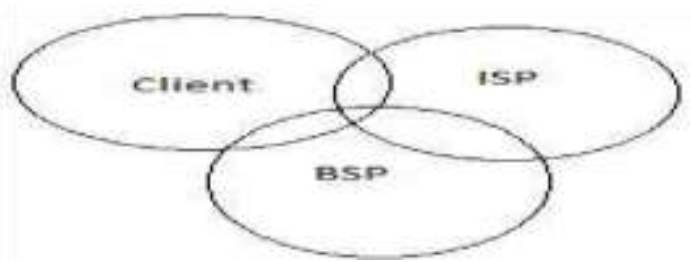

Figure 5. Simple view of system

\section{B. Performance Comparison}

Using our resource renting scheme, temporary services are rented for all requests whose waiting time are equal to the deadline. Which can guarantee that all requests are served with better service quality. Hence, our scheme is superior to the traditional resource renting scheme in the terms of service quality. Next, we conduct a series of calculations to compare the profit obtained by the DQG renting scheme with the SQU renting scheme. In proposed system we are using the Double-Quality-Guaranteed (called DQG) renting scheme that can achieve more profit compared to the compared Single-Quality-Unguaranteed (called SQU) renting scheme in the premise of completely guaranteeing the service qualityis maximized. 


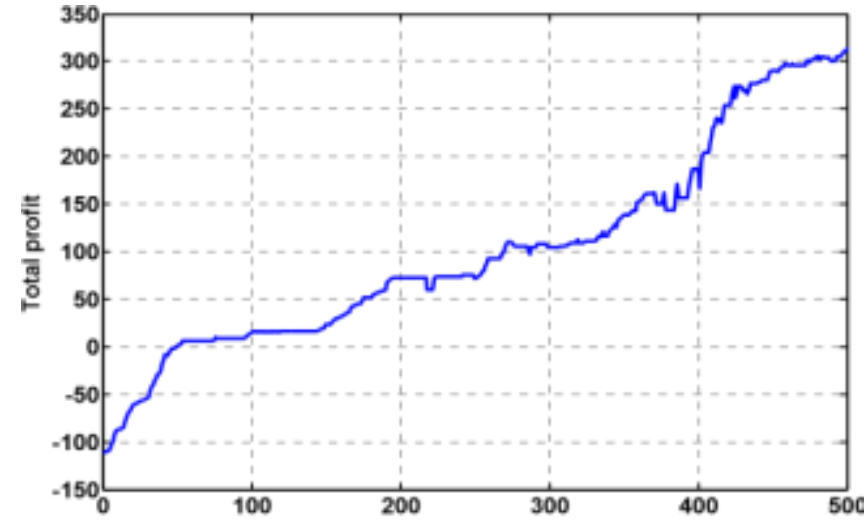

Figure 6. An example of Resource Consolidation Improvement in case of a poor initial solution.

The upper bound profit in this case is 2000. It can be seen that the resource consolidation method based on the force-directed search gradually increases the total profit. Also some decrease in the total power dissipation can be observed, which is due to the nature of the force-directed search.

Figure 7 shows the average run time of the proposed heuristic for different number of clients and servers. Although the average number of servers for the second scenario is double this number for the first scenario, the run time does not increase a lot because the force-directed search is based on the server types not the actual servers. It can be seen that in case of having an average of 400 client-tiers and 1000 servers, the solution is found in less than 1.5 minutes, which is acceptable for cases with decision epoch lengths in the order of half an hour.

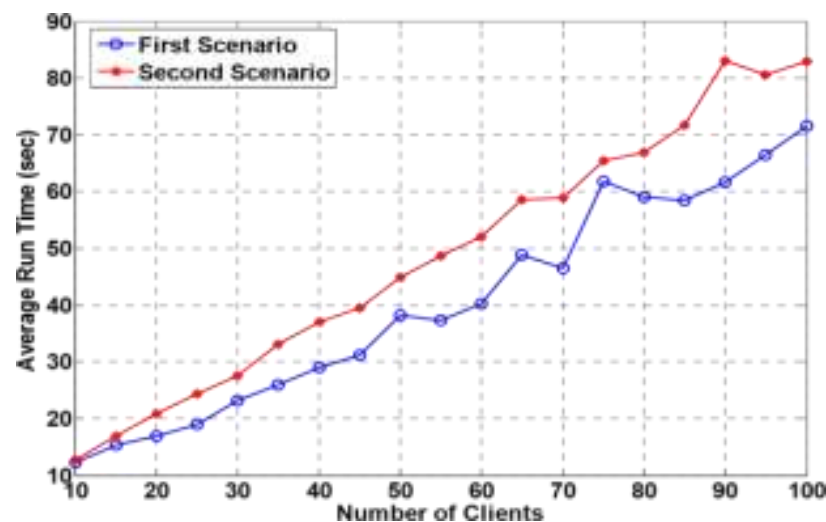

Figure 7. Average run time of FRA algorithm on 2.8GHZ E5550 server (Intel) for different number of clients.

To show the characteristic of the proposed solution, Figure 8 shows the average ratio of / for the clients with the Gold SLA class for different ratios of / . As expected, the ratio of the EPT arrival rate is increased to compensate increase in the penalty value. For some penalty values, the EPT arrival rate is more than the contract arrival rate which is due to the iterative nature of the resource allocation in the case of the Gold SLA.

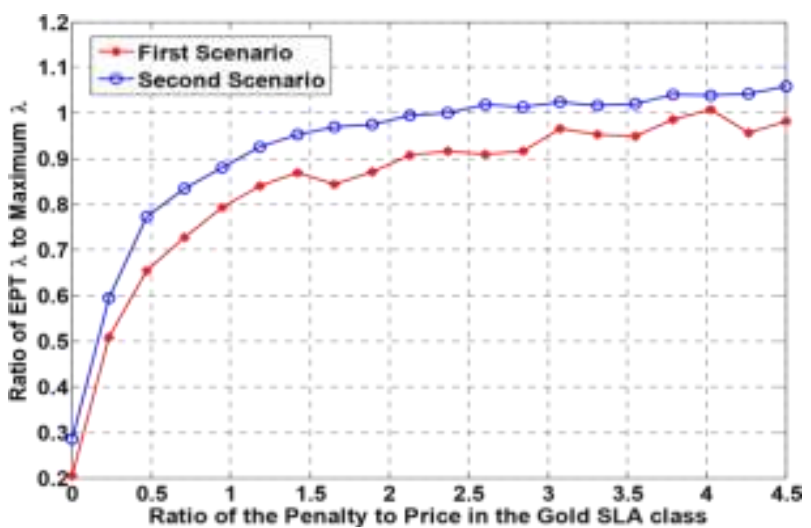

Figure 8. Ratio of EPT inter-arrival rate to maximum inter-arrival rate for different penalty values for Gold SLA class. 
Figure 9 shows the average utilization factor of the servers in case of different / values. Lowering the value of / means that the idle energy cost accounts for a bigger portion of the total energy cost in case of full utilization, which tends to result in more consolidation in servers and thus fewer ON servers.

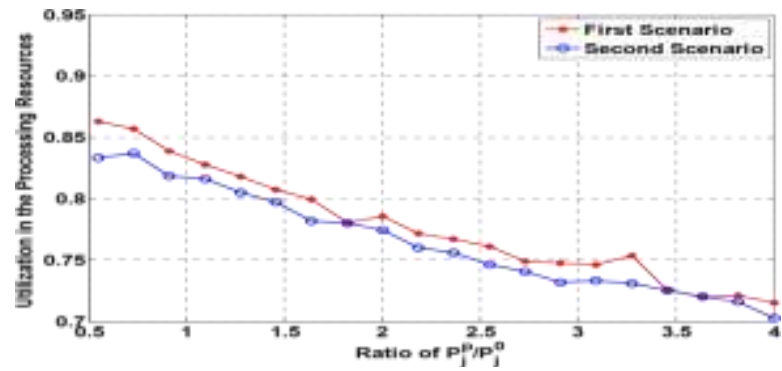

Figure 9. Utilization of the servers for different values.

\section{Conclusion}

A new approach called Double Quality Guaranteed renting method for service providers is proposed to ensure the quality of service demands as well as to enhance the profit. To minimize the wastage of resources, this method includes both the short term renting method as well as long term renting method. To vary the system sizes, a queuing model is implemented for multi-server system. The data hosting algorithm is implemented before the DQG method, to make the suitable choice of cloud as well as to enhance the profit of service provider. After that, an issue of optimal configuration of profit increasing is defined in that several aspects are determined. This proposed double renting strategy is implemented to heterogeneous cloud environment.

\section{References}

[1]. Jing Mei, Kenli Li, Member, Aijia Ouyang and Keqin Li, Fellow, 2015 , A Profit Maximization Scheme with Guaranteed Quality of Service in Cloud Computing

[2]. G. P. Cachon and P. Feldman, 2010. Dynamic versus static pricing in the presence of strategic consumers.

[3]. J. Cao, K. Hwang, K. Li, and A. Y. Zomaya, , 2013. Optimal multi-server configuration for profit maximization in cloud computing.

[4]. A. Fox, R. Griffith, A. Joseph, R. Katz, A. Konwinski,G. Lee, D. Patterson, A. Rabkin, and I. Stoica, 2009. Above the clouds: A berkeley view of cloud computing.

[5]. R. Buyya, C. S. Yeo, S. Venugopal, J. Broberg, and I.Brandic, 2009. Cloud computing and emerging it platforms:Vision, hype, and reality for delivering computing as the 5 th utility.

[6]. P. Mell and T. Grance, 2009. The NIST definition of cloud computing.National institute of standards and technology Information Technology Laboratory.

[7]. J. Chen, C. Wang, B. B. Zhou, L. Sun, Y. C. Lee, andA. Y. Zomaya, 2011.Tradeoffs between profit and customer satisfaction for service provisioning in the cloud.

[8]. J. Mei, K. Li, J. Hu, S. Yin, and E. H.-M. Sha, 2013. Energy aware preemptive scheduling algorithm for sporadic tasks on dvs platform.

[9]. P. de Langen and B. Juurlink, 2009.Leakage-aware multi processor scheduling.

[10]. S. Liu, S. Ren, G. Quan, M. Zhao, and S. Ren, 2013. Profit aware load balancing for distributed cloud data centers.

[11]. Z. Liu, S. Wang, Q. Sun, H. Zou, and F. Yang, , 2013. Cost-aware cloud service request scheduling for saas providers.

\section{Authors:}

Sowmya Bhaskarla is a student of Computer Science \& Engineering from QIS Institute of Technology, She

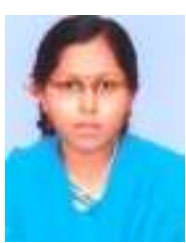
Presently pursuing M.Tech (CSE) in this college. She received B.Tech from JNTUK in the year of 2013.

R Lakshmi Tulasi is a Professor, H.O.D of QIS Institute of Technology, Ongole. She received M.Tech from

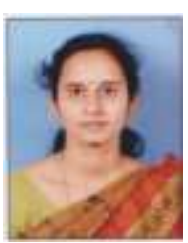
JNTUCEA. She is pursuing Ph.D. at JNTUH. She is a good Researcher in semantic web, Computer Networks. She attended Various National and International Workshops and Conferences. 